\title{
Technique of Semantic Unambiguity for a Concept Selection of Terms in Focused Contexts with Reinforcement Learning Integration
}

\author{
Churee Techawut \\ Computer Science Department, Faculty of Science \\ Chiang Mai University \\ Chiang Mai, Thailand \\ Email: churee.t [AT] cmu.ac.th
}

\author{
Khananat Jaroenchai \\ Computer Science Department, Faculty of Science \\ Chiang Mai University \\ Chiang Mai, Thailand \\ Email: khananat09 [AT] gmail.com
}

\begin{abstract}
Nowadays, there have been many developments of learning processes for computers to understand the meaning of words and their semantic similarities in order for the computers to better communicate, interact and exchange information with humans. Semantic learning development is a major issue because computers cannot comprehend the suitable meaning of words in the concerning concept. As a result, this research is proposing and exploring the efficiency of the technique of semantic unambiguity in order to clarify the Term Concepts in the focused contexts. From the case study with 22 contexts, 62 term, and 475 synsets, it was shown that Reinforcement Learning could accurately select the suitable term concepts for the focused contexts, with Precision $=\mathbf{0 . 7 7 5 6}$, Recall $=\mathbf{0 . 7 7 5 6}$ and $\mathrm{F}$-Measure $=\mathbf{0 . 7 7 3 5}$. Therefore, it can be concluded that the Technique of Semantic Unambiguity for a Concept Selection of Terms in Focused Contexts has high accuracy when applying the Reinforcement Learning.
\end{abstract}

Keywords: Semantic Unambiguity; Concept Selection; Natural Language Processing; Reinforcement Learning; Knowledge Engineering

\section{INTRODUCTION}

For years, computer research scientists have been developing learning processes for computers to learn and understand the human nature in order to better respond to human needs through human languages. Many problems have been continuously examined, analyzed and fixed, including the variety of natural languages and the complexity of language structure.

At present, there are many learning processes for computers to understand the meaning of words and their semantic similarities [1][2], tracking fake information [3] or their positive and negative connotations [4] for more accurate communication and more convenient information exchange with humans; for example, a computer-assisted translation software, an Information Retrieval System which can retrieve words with similar meanings, etc.

After a while of conducting this research, the researcher came across a problem regarding the categorization of terms for ontology structuring [5] because the concept used by the system misrepresented the meaning of terms in the focused context for determining the semantic similarities scores; therefore, generating many incorrect results. However, from the study, it was found that the technique for concept selection suitable for each term has led to more accuracy in categorization.

This research further studied the synset detection process by [5] to increase the efficiency of synset detection process. The problem was encountered when the evaluated terms had only few related concepts. For example, there was only one concept for the term meaning, making this one concept the main term for other semantic search of other terms. Therefore, the final result was unsuitable for the focused context. Problem solving process development [5] was improved to create the Technique of Semantic Unambiguity for a Concept Selection of Terms in Focused Contexts to truly identify the meaning of each word under the same context and categorize these words to create semi-automatic ontology [5] by using Precision, Recall and F-Measure to compare the efficiency with the synset detection process.

This paper was divided into section as follows: Section 2 presented knowledge involving with this research, demonstrating the importance, the concepts and the guideline for the development and the improvement of synset detection process to be more effective. Section 3 presented the synset detection process used to solve problems. This Synset Detection Process was improved to be more effective for semantic identification under the focused contexts and other terms. Section 4 presented the process and the variables used to evaluate the efficiency of the synset detection process. Section 5 discussed the results of the study; and Section 6 summarized the results of the study and presented the recommendation from the researcher.

\section{LITERATURE REVIEW}

This section explains about background knowledge and techniques used in this research 


\section{A. Background knowledge}

1) Glossary is elucidation the meaning of terms that are appropriate or desirable with context. It is one of the process in Analysis Workflow of Unified Process for ONtology building (UPON) process [6]. Normally, Knowledge Engineers are the ones who define the meaning of each term for mutual understanding of ontology users because some terms may have several meanings; for example "Guard" can be a player position in sports, or a person who watches over some things, or other meanings.

\section{B. Related works}

Semantic Similarity and Communication Context are important for language translation. Normally, for accurate and precise communication, words and sentences used in communication should have only one meaning. However, the variety in several aspects creates the ability to apply one word in several contexts, leading to one word having several meanings. Therefore, a context is a crucial part to minimize the different meanings of each word for more accurate interpretation; for example, "Guard" in a context of a profession and "Guard" in a context of a player position in sports. One of the things that can be used to set the context of the words is the vocabulary set used in one sentence [2] or words that can be seen in the same document, table or context [5]. The related works of how to interpret the vocabulary by its context are as follows:

1) Structure Vector Space (SVS) Model [2] is model presents how to interpret the vocabulary using verbs and nouns in a sentence by creating Lemma Meaning to show the relationships between verbs and nouns combined to form a Vector network and using Lemma Meaning as a foundation for the decision making process to evaluate the vocabulary.

2) Machine Learning Based Senti-word Lexicon (MLBSL) [9] is a technique used to categorize vocabulary in each context based on their positive and negative connotations. The Machine Learning Technique has two main evaluation processes which are:

- Data Preparation: The sentences will be aligned and ready for evaluation.

- Lexicon Forming: The data set from data preparation will be evaluated in order to train SVM, and this SVM will be further evaluated for categorization.

3) Synset Detection [5] is the selection process assigning the synset suitable with the focused context to each term. The Reinforcement Learning technique [10] is used to select synset from the vocabulary list on the same multi-dimension data table, or from under the same column. The Semantic Similarity Score (SSS) is then used as an accumulative value to further determine the possibility of the synset suitable with the focused context.

\section{PROBLEM SOLVING}

Figure 1 shows a process overview divided into 3 parts.

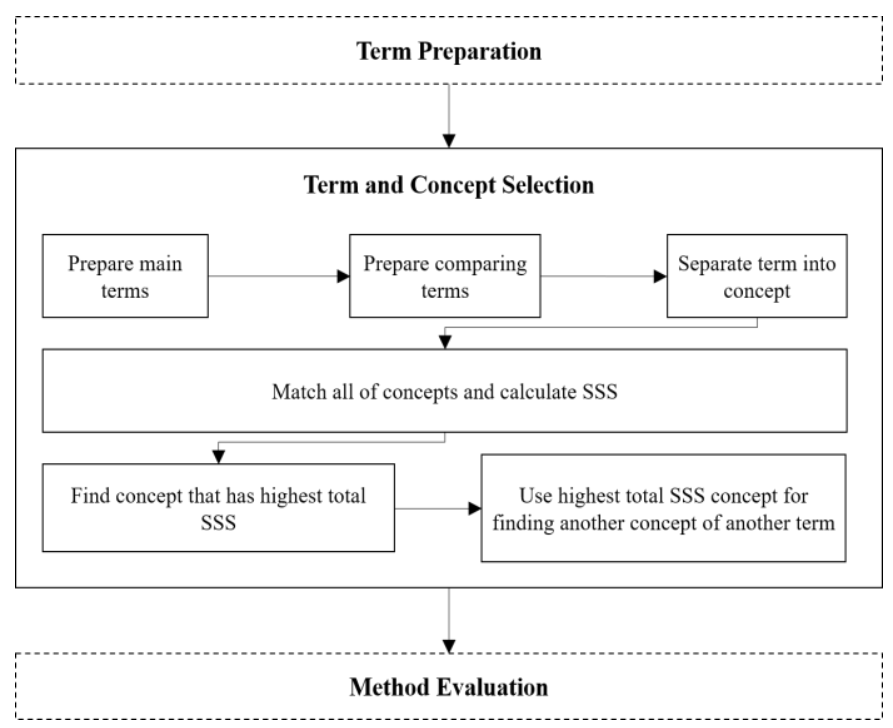

Figure 1 Process overview

\section{A. Term preparation}

Terms $(T)$ were prepared by putting in predetermined loops. Each loop contained at least 3 terms ( $T i$ when $i \leq n$ and $n \geq 3$ ), the first term going in was called $T 1$, the second term going in was called $T 2$, and so forth.

\section{B. Term concept selection}

Terms (T) were prepared by putting in predetermined loops. Each loop contained at least 3 terms (Ti when $\mathrm{i} \leq \mathrm{n}$ and $\mathrm{n}$ $\geq 3$ ), the first term going in was called $\mathrm{T} 1$, the second term going in was called T2, and so forth.

1) Main term preparation: Out of several terms in a loop, the system would assign one main term to be compared with other terms. The first main term was the first term in the loop. After the first main term was compared for semantic similarity with all other compare terms, the system would move on to set the second term to be the second main term and start the comparison for semantic similarity with all other compare terms, and so forth.

2) Compare term preparation: Compare terms were the terms that would be compared with the main term. The system would assign the term next to the main term to be the first compare term, and the second compare term was the term next to the first compare term, and so forth. After the system was done with the comparison for semantic similarity, the main term would be changed. Steps 1) and 2) are shown in Figure 2. 


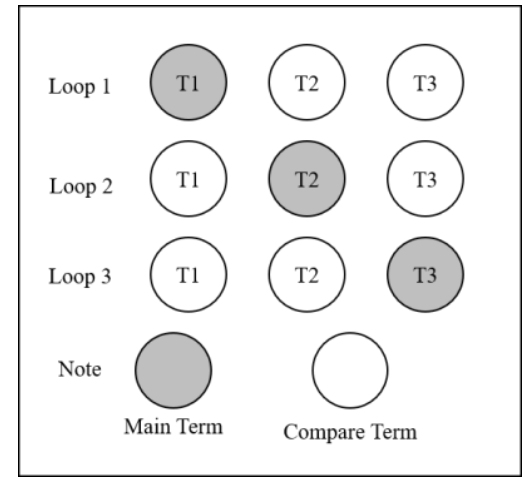

Figure 2 Term preparation

3) Main term and compare term separation into concept: During this stage, the system would operate based on the WordNet [7] Vocabulary Database by pulling all synsets of both main terms and compare terms as each Concept ( $\mathrm{TiCj}$ ) and compare them with each concept in order to find the semantic similarity of every concept $(\mathrm{Si})$. An example is shown in Figure 3.

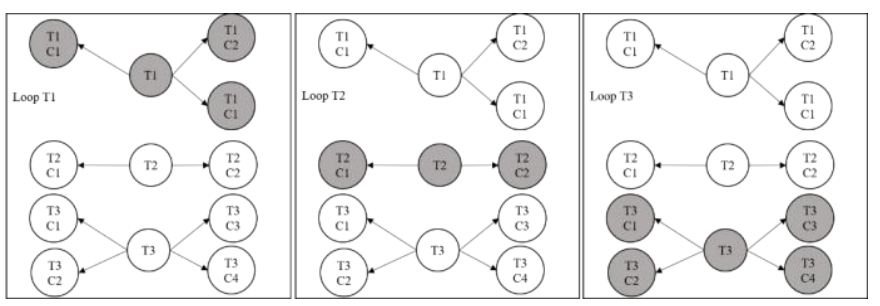

Figure 3 Separating terms into concepts

4) Semantic Similarity Score Measurement between main term concepts and compare term concepts: The system would put the pair concept of one main term and one compare term into the Wu and Palmer [8] Equation as shown in Figure 4. The received semantic similarity score ( $\mathrm{Si}$ ) would be stored in the same manner as the Q-table as displayed in Eq. (1) for future comparison. (The example in Figure 4 examined the semantic similarities of $\mathrm{T} 1$ and $\mathrm{T} 2$ without having to compare $\mathrm{T} 1$ to $\mathrm{T} 3$ and $\mathrm{T} 2$ to $\mathrm{T} 3$ in the future).

$$
\mathrm{Q}(\mathrm{Si}, A j)=\sum_{j=0}^{n} \operatorname{Max}(\operatorname{Sim}(S i, T j))
$$

$\mathrm{Q}$ is the function used to calculate for the reward score of concept $\mathrm{i}$. Si is the State, or the Concept of the current focused term. A is the action, or the act of determining the SSS with other terms under the same context with the focused concept. Tj is other terms under the same context as term of $\mathrm{S}_{\mathrm{i}}$. Sim is the function used to calculate for the SSS value.

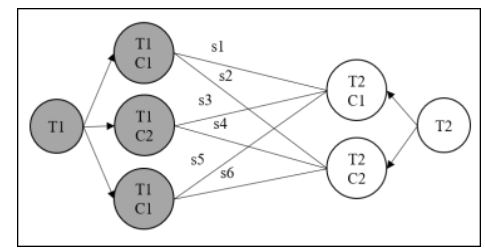

Figure 4 Calculating semantic similarity score

5) Winner Concept assignment: Winner Concept is the concept with the highest value of Semantic Similarity Score. After semantic similarity score measurement, the semantic similarity score of each paired concept, with threshold at $\mathrm{Si} \geq$ 0.7 , would be set as a score for that concept. Setting threshold at 0.7 would filter out more concepts unrelated to the focused context than setting threshold at 0.5 , and the remaining concepts could be evaluated to find the winning concept appropriately. After that, the system would calculate the total score for each concept, and the concept with the highest total score would be assigned the Winner Concept of that particular focused context. The total scores of all 3 terms were shown in Figure 5.

\begin{tabular}{|ll|}
\hline $\mathrm{T} 1 \mathrm{C} 1->\mathrm{T} 2 \mathrm{C} 2, \mathrm{~T} 3 \mathrm{C} 1, \mathrm{~T} 3 \mathrm{C} 4=0.72+0.75+0.80$ & $=2.27$ Winner \\
$\mathrm{T} 1 \mathrm{C} 2->\mathrm{T} 2 \mathrm{C} 1, \mathrm{~T} 3 \mathrm{C} 2=0.72+0.71$ & $=1.43$ \\
$\mathrm{~T} 1 \mathrm{C} 3->\mathrm{T} 3 \mathrm{C} 3=0.70$ & $=0.70$ \\
$\mathrm{~T} 2 \mathrm{C} 1->\mathrm{T} 1 \mathrm{C} 2=0.72$ & $=0.72$ \\
$\mathrm{~T} 2 \mathrm{C} 2->\mathrm{T} 1 \mathrm{C} 1, \mathrm{~T} 3 \mathrm{C} 1=0.72+0.74$ & $=1.46$ \\
$\mathrm{~T} 3 \mathrm{C} 1->\mathrm{T} 1 \mathrm{C} 1, \mathrm{~T} 2 \mathrm{C} 2=0.75+0.74$ & $=1.49$ \\
$\mathrm{~T} 3 \mathrm{C} 2->\mathrm{T} 1 \mathrm{C} 2=0.71$ & $=0.71$ \\
$\mathrm{~T} 3 \mathrm{C} 3->\mathrm{T} 1 \mathrm{C} 3=0.70$ & $=0.70$ \\
$\mathrm{~T} 3 \mathrm{C} 4->\mathrm{T} 1 \mathrm{C} 1, \mathrm{~T} 2 \mathrm{C} 2=0.8+0.74$ & $=1.54$
\end{tabular}

Figure 5 Summarizing semantic similarity scores and assigning winner concept.

6) Follower Concept assignment: Follower Concept is the concept with the closest semantic similarity to the Winner Concept. After assigning the Winner Concept, the system would search that concept for the semantic similarity score for the Follower Concept. The concept with second highest semantic similarity score of other term concept, compared to the main term's concept, would be assigned the concept related to the focused context of the terms in this loop. This comparison would be conducted until all terms were assigned their own concepts. Example in Figure 6 shows that how winner of $\mathrm{T} 1$ find Follower from $\mathrm{T} 2$ and $\mathrm{T} 3$

After assigning a set of the Winner Concept and the Follower Concepts presenting the meaning of terms in the focused context, this concept set would be further evaluated and compared with the answer keys as shown in section 4 . 


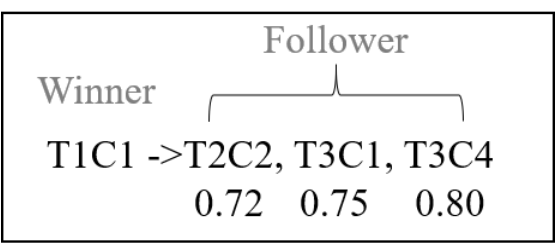

Figure 6 Assigning follower concepts.

\section{Evaluation}

\section{A. Evaluation objective}

The objective of the evaluation is to value the efficiency of the semantic unambiguity technique in assigning concepts which are suitable with the focused context using Precision, Recall and F-Measure.

\section{B. Variable}

\section{1) Dependent variable}

- Precision is the precision of the term concept selection from the semantic unambiguity technique. The technique is deemed very efficient when the Precision value is near 1 .

- Recall is the efficiency of the term concept selection from the focused semantic unambiguity technique. The technique is deemed very efficient when the Recall value is near 1 .

- F-Measure is calculated with Precision Value and Recall Value. It is used to present the overall efficiency of the term concept selection from the semantic unambiguity technique. The technique is deemed very efficient when the F-Measure value is near 1.

2) Independent variable

- True Positive (TP) is the number of terms that the Agent selects the concepts that match with the answer key, with the answer key being the concepts from the Positive concept set.

- False Positive (FP) is the number of terms that the Agent selects the concepts that does not match with the answer key, with the answer key being the Positive concept set but the Agent selects the concepts from the Negative concept set.

- False Negative (FN) is the number of terms that the Agent selects the concepts that does not match with the answer key, with the answer key being the Negative concept set but the Agent selects the concepts from the Positive concept set.

- True Negative (TN) is the number of terms that the Agent selects the concepts that match with the answer key, with the answer key being the concepts from the Negative concept set.

The positive concepts are concepts in a term concept set with the meaning related to the focused context of efficiency evaluation; whereas, the negative concepts are concepts in a term concept set with the meaning related to other contexts, either one with the previous efficiency evaluation result, or one without the efficiency evaluation result yet, waiting to be evaluated, or even not related to the experimented at all.

There are two groups of datasets used in the experiment. The first experiment contains 7 contexts as follows: (1) player positions in Basketball (2) professions1 (3) player positions in Football (4) cooking methods (5) sports equipment (6) mammals1 and (7) seven religious sins. Each context may contain the same terms as the others do, such as "Guard" which can be found in (1), (2) and (5). However, the meaning of the term is different according to each context.

The second experiment uses enlarged dataset by adding 15 contexts to the first experiment as listed: (8) military term (9) mammals2 (10) fruit1 (11) color1 (12) fruit2 (13) color2 (14) color3 (15) fruit3 (16) climate (17) symptom (18) professions2 (19) nature thing (20) personal definition (21) computer tech term (22) direction term.

These 22 contexts has 62 terms and 475 synsets are chosen because they contain the same terms with different meanings in different contexts. The overall terms used in this experiment are shown in Table 1 - 4 .

TABLE 1 Displaying terms used in this experiment (context $1-6$ )

\begin{tabular}{cccccc} 
(1) & (2) & $(\mathbf{3})$ & (4) & (5) & (6) \\
\hline Guard & Guard & Center & Steam & Guard & Bat \\
Forward & Cook & Forward & Smoke & Stud & Whale \\
Center & Guide & Back & Roast & Racket & Sloth \\
& & Goalkeeper & Bake & Bat & Dog \\
& & Striker & Fried & &
\end{tabular}

TABLE 2 Displaying terms used in this experiment (context 7 - 12)

\begin{tabular}{cccccc}
\hline $\mathbf{( 7 )}$ & $(\mathbf{8})$ & $\mathbf{( 9 )}$ & $\mathbf{( 1 0 )}$ & $\mathbf{( 1 1 )}$ & $\mathbf{( 1 2 )}$ \\
\hline Lust & Guard & Bat & Apple & Red & Peach \\
Gluttony & Attack & Pig & Cherry & Green & Guava \\
Greed & Escape & Cow & Melon & Blue & Grape \\
Sloth & Spy & & Orange & Orange & Apple \\
Wrath & & & Lime & & \\
Envy & & & & & \\
Pride & & & & & \\
\hline
\end{tabular}

TABLE 3 Displaying terms used in this experiment (context $13-18$ )

\begin{tabular}{cccccc}
\hline $\mathbf{( 1 3 )}$ & $\mathbf{( 1 4 )}$ & $\mathbf{( 1 5 )}$ & $\mathbf{( 1 6 )}$ & $\mathbf{( 1 7 )}$ & $\mathbf{( 1 8 )}$ \\
\hline Peach & Peach & Peach & Cold & Cold & Singer \\
Blue & Red & Orange & Hot & Crick & Farmer \\
Orange & Green & Apple & Rain & Convulsion & Driver \\
& & & & Depression \\
& & & Anemia \\
\hline
\end{tabular}

TABLE 4 Displaying terms used in this experiment (context 19-22)

\begin{tabular}{cccc}
\hline $\mathbf{( 1 9 )}$ & $\mathbf{( 2 0 )}$ & $\mathbf{( 2 1 )}$ & $\mathbf{( 2 2 )}$ \\
\hline Nature & Star & Driver & Right \\
Star & Actor & Network & Left \\
Tree & Player & Hardware & Back \\
& & & Above
\end{tabular}




\section{Hypothesis}

The hypothesis of this evaluation is that, if Precision, Recall and F-Measure of term concept selection from the semantic unambiguity technique are over 0.7 , the efficiency of the semantic unambiguity technique to select the term concept with the meaning suitable with the focused context is very well.

\section{Experimental procedure}

a) Prepare a term list by dividing term sets according to the focused contexts, with each term set containing at least 3 terms.

b) Prepare the proper answer key for each term in the focused contexts. The answer keys are concept sets with the meanings that match with the focused contexts; for example, "Guard" in the context of a player position in sports means (1) a player position in sports such as Basketball, Hockey, etc. (2) a person in a player position called Guard in Basketball, Hockey, etc.

c) Evaluate the term lists from the semantic unambiguity technique by comparing them with the answer keys to determine the accuracy of the selected concepts. If the selected concept contains more than 1 term, all terms will be re-evaluated. If the result matches with the answer key, each concept will gain 1 efficiency score. If the result does not match with the answer key, the concept will receive no score.

d) Process the final results and create the Confusion Matrix Table to calculate Precision, Recall and Fmeasure for each focused context.

e) Repeat step (d) for every focused context and find the average Precision, Recall and F-measure for all focused contexts.

\section{Evaluation Discussion}

The semantic unambiguity technique for concept selection using the reinforcement learning technique by setting a threshold to determine appropriate term concept pairs for semantic similarity provided the highest accumulated semantic similarity scores at Precision $=0.8788$, Recall $=0.9667$ and Fmeasure $=0.9206$ in the first experiment. In addition, it is possible to have decreasing efficacy value while having a large dataset. However, the second experiment still provided a high value of efficacy at Precision $=0.7756$, Recall $=0.7756$ and F-measure $=0.7735$.

On the contrary, the traditional method, which was the semantic unambiguity technique for concept selection using the reinforcement learning technique "without" setting a threshold for semantic similarity, provided Precision $=0.8$, Recall $=0.9655$ and F-measure $=0.875$ in the first experiment. However, this method provided a low value of efficacy with Precision $=0.3731$, Recall $=0.3912$ and Fmeasure $=0.3735$ when using enlarge dataset in the second experiment. The efficacy comparisons of the first and second experiments are shown in Figure 7.

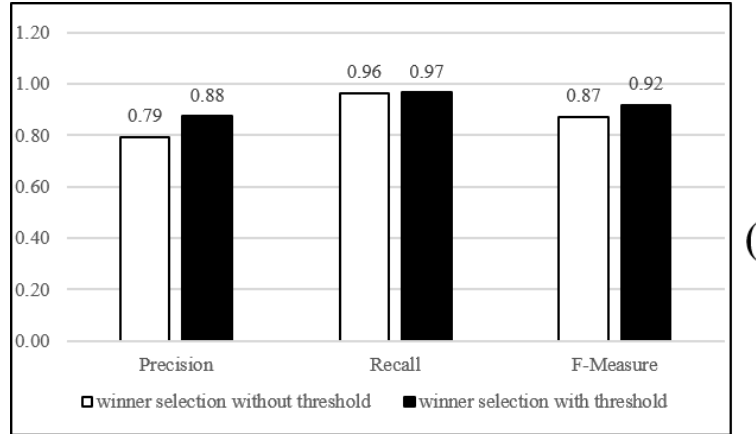

(a)

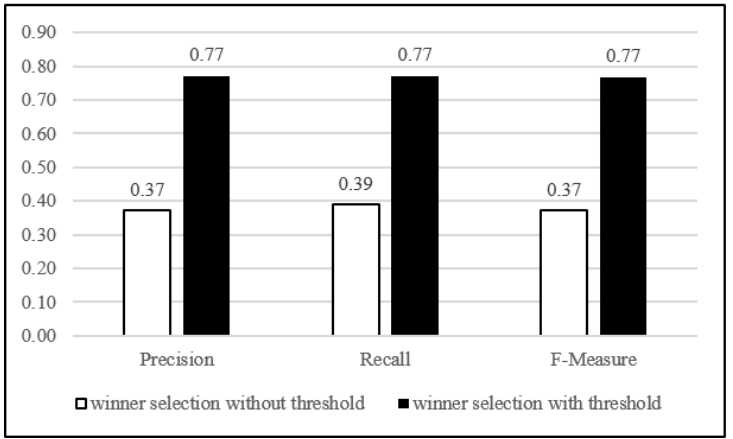

(b)

Figure 7 Graph showing efficiency scores "with" and "without" threshold of semantic similarity score for concept selection in the (a) first experiment (7 contexts) and (b) second experiment (22 contexts)

From the experiment, it was found that the semantic unambiguity technique for concept selection using the reinforcement learning technique was considered effective for term concept selection to fit the focused context. The semantic unambiguity technique for concept selection using the reinforcement learning technique by setting a threshold as a score filter to determine appropriate term concept pairs was more efficient than the method without the threshold, technique for this technique for this experiment. This was due to the fact that the method setting the threshold for term concept pairs could filter out the extremely low semantic similarity score. As can be seen in some cases that the term concepts gained the highest total score by including the semantic similarity score less than 0.7 , having more number of members, as shown in the "cooking method" context in Figure 8. Without the threshold, this concept was automatically chosen due to its highest score for each term concept comparison. However, the main term and the compare term would not be suitable for the focused knowledge. With the threshold setting, the concept with little to no relation would be excluded. This research used 0.7 score as an appropriate standard threshold for more efficient results. The comparison according to the context of term concepts regarding Precision was shown in Figure 8(a) and F-Measure was shown in Figure 8(b).

Another possible cause for different efficiency scores for the "cooking method" context was the SSS from Wu and Palmer's calculation. The semantic unambiguity technique for concept with the meaning "cooked by frying in fat" for the term "Fried", which was the Winner Concept for the semantic unambiguity technique, was used to find the Follower Concept 
from the term "Steam". It was found that the concept of term "Steam" with SSS at 0.4, the highest score for this scenario, was granted concept "travel by means of steam power" and "emit steam". Theoretically, the researcher expected that the highest SSS of the concept "Fried" should be the concept "cook something by letting steam pass over it" or "water at boiling temperature diffused in the atmosphere" of term "Steam", with SSS only at 0.28 and 0.2 respectively as shown in Table 5 compare with Table 6 . As can be seen that the efficiency score of the semantic unambiguity technique for concept selection using the reinforcement learning technique by inserting all concept pairs into the mix generated less scores compared with the method which included only suitable term concept groups into the system. With threshold setting, the low SSS would be filtered out of the system, changing the Winner Concept of term "Steam" to "cook something by letting steam pass over it", and consequently yielded other Follower Concepts more appropriately as shown in Figure 8.

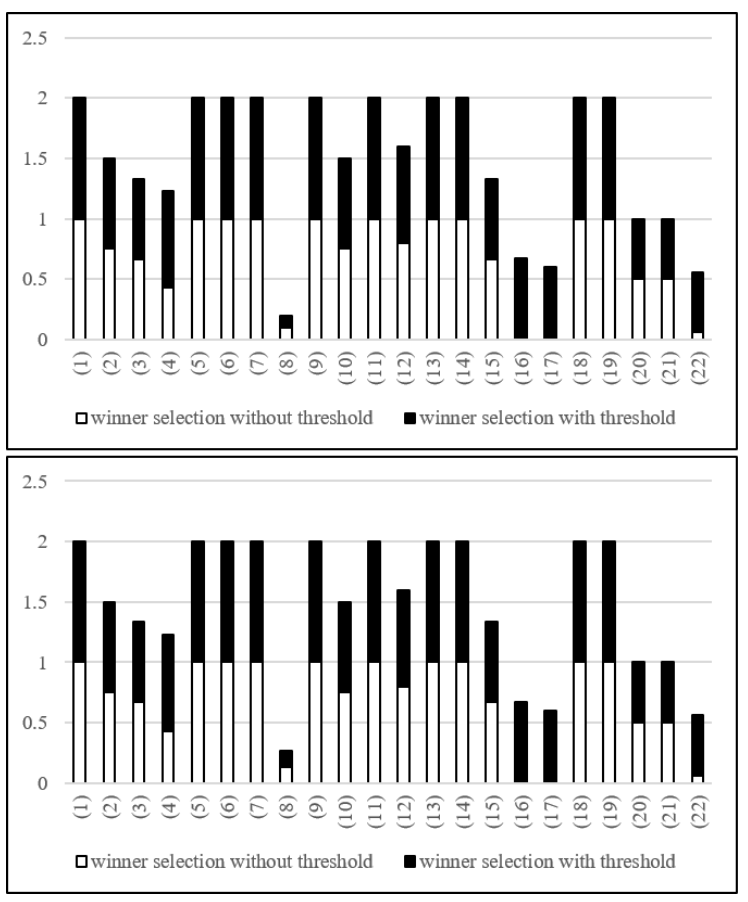

(a)

Figure 8 Bar chart showing (a) Precisions and (b) F-Measure for each context.

TABLE 5 Selected concepts without threshold filtering (Winner concepts generated from semantic unambiguity technique).

\begin{tabular}{|c|c|r|}
\hline \multicolumn{2}{|c|}{ Without threshold filtering } \\
\hline Term & Concept meaning & $\begin{array}{r}\text { SSS } \\
\text { with } \\
\text { winner }\end{array}$ \\
\hline Steam & travel by means of steam power & 0.4 \\
\hline Steam & emit steam & 0.4 \\
\hline Roast & meat cooked by dry heat in an oven & 0.5 \\
\hline Bake & be very hot due to hot weather or exposure to the sun & 0.4 \\
\hline Smoke & inhale and exhale smoke from cigarettes cigars pipes & 0.25 \\
\hline Smoke & emit a cloud of fine particles & 0.25 \\
\hline Fried* & cooked by frying in fat & Winner \\
\hline
\end{tabular}

TABLE 6 Selected concepts with threshold filtering (Winner concepts generated from semantic unambiguity technique).

\begin{tabular}{|c|c|r|}
\hline \multicolumn{2}{|c|}{ Threshold filtering } \\
\hline Term & Concept meaning & $\begin{array}{c}\text { SSS } \\
\text { with } \\
\text { winner }\end{array}$ \\
\hline Steam* & cook something by letting steam pass over it & Winner \\
\hline Roast & cook with dry heat usually in an oven & 0.8 \\
\hline Bake & cook and make edible by putting in a hot oven & 0.8 \\
\hline Smoke & $\begin{array}{c}\text { inhale and exhale smoke from cigarettes cigars } \\
\text { pipes }\end{array}$ & 0.25 \\
\hline Fried & cooked by frying in fat & 0.28 \\
\hline
\end{tabular}

\section{SUMMARY AND RECOMMENDATION}

After setting a threshold to the semantic similarity scores during concept selection searching for the concept with the highest semantic similarity accumulated scores, Precision, Recall and F-measure all increased compared to the traditional method of merely using the highest semantic similarity scores and this experiment shown more difference efficacy between setting a threshold and traditional method when add more contexts in experiment. This was due to the fact that some terms had only one concept and, as a result, gained the highest accumulated score. Even when the similarity scores from the paired term was low, after combining with other terms, the term with one concept always ended up being the one with the highest total score. After threshold setting, the number of concepts with semantic similarity scores lower than 0.7 were eliminated and only terms with strong relations were included in the calculation.

The limitation for this study was that the evaluation ability. The technique of semantic unambiguity could only be conducted with terms with meaning existed in WordNet Database. Compound words or any proper nouns what were not included in the WordNet Vocabulary Database could not be evaluated.

The technique of semantic unambiguity for concept selection of terms in focused contexts can offer the appropriate meanings for terms in the focused contexts by using other terms in the same context to search for the concepts with the most fitting semantic relations. It is particularly suitable for (1) term categorization in knowledge management (2) a semantic suitability selection technique under focused contexts regarding natural languages, as can be seen in Figure 9. 


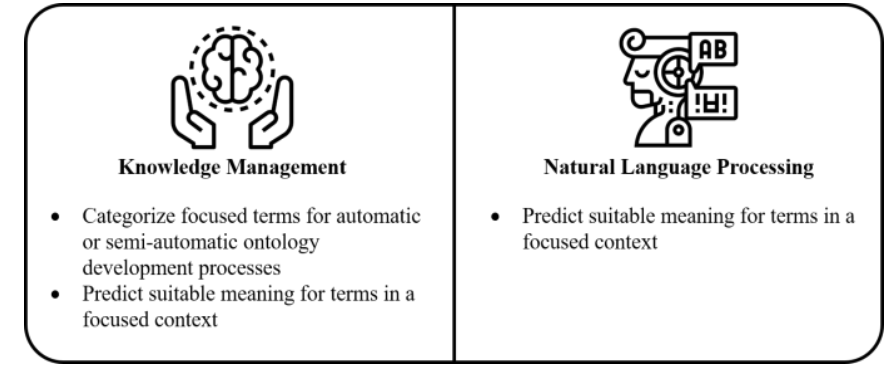

Figure 9 Examples of various implementation of semantic unambiguity technique.

\section{ACKNOWLEDGMENT}

This research was funded by Faculty of Science, Chiang Mai University (R000022443).

\section{REFERENCES}

[1] E. Kotzé, B.A. Senekal, and W. Daelemans, Automatic classification of social media reports on violent incidents in South Africa using machine learning. South African Journal of Science, 116(3-4), 1-8, 2020

[2] K. Erk and S. Padó, A structured vector space model for word meaning in context. In Proceedings of the 2008 Conference on Empirical Methods in Natural Language Processing (pp. 897-906), 2008.
[3] P. Hajek, A. Barushka, and M. Munk, Fake consumer review detection using deep neural networks integrating word embeddings and emotion mining. Neural Computing and Applications, 32(23), 17259-17274, 2020

[4] S. M. Mohammad and P. D. Turney, Crowdsourcing a word-emotion association lexicon. Computational intelligence, 29(3), 436-465, 2013.

[5] K. Jaroenchai and C. Techawut, "Know-Ont Based Ontology Modeling Approach for Skill Knowledge Extraction," International Journal of Knowledge Engineering vol. 4, no. 2, pp. 81-86, 2018.

[6] A. De Nicola, M. Missikoff and R. Navigli, A software engineering approach to ontology building. Information systems, 34(2), 258-275, 2009.

[7] G. A. Miller, WordNet: An electronic lexical database. MIT press, 1998.

[8] C. Corley and R. Mihalcea, Measuring the semantic similarity of texts. In Proceedings of the ACL workshop on empirical modeling of semantic equivalence and entailment (pp. 13-18). Association for Computational Linguistics, 2005.

[9] A. Hamouda, M. Marei and M. Rohaim, Building machine learning based senti-word lexicon for sentiment analysis. Journal of advances in information technology, 2(4), 199-203, 2011.

[10] L. P. Kaelbling, M. L. Littman and A. W. Moore, Reinforcement learning: A survey. Journal of artificial intelligence research, 4, 237-285, 1996. 